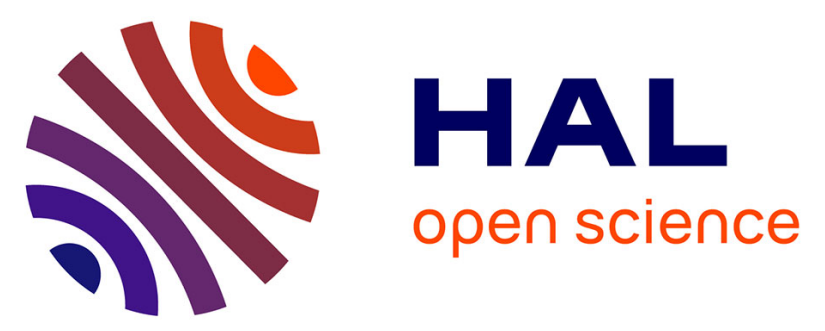

\title{
Material Decomposition in X-ray Spectral CT Using Multiple Constraints in Image Domain
}

Bingqing Xie, Ting Su, Valerie Kaftandjian, Pei Niu, Feng Yang, Marc C Robini, Yuemin Zhu, Philippe Duvauchelle

\section{- To cite this version:}

Bingqing Xie, Ting Su, Valerie Kaftandjian, Pei Niu, Feng Yang, et al.. Material Decomposition in Xray Spectral CT Using Multiple Constraints in Image Domain. Journal of Nondestructive Evaluation, 2019, 38 (1), 10.1007/s10921-018-0551-8 . hal-01978570

\author{
HAL Id: hal-01978570 \\ https://hal.science/hal-01978570
}

Submitted on 18 Nov 2021

HAL is a multi-disciplinary open access archive for the deposit and dissemination of scientific research documents, whether they are published or not. The documents may come from teaching and research institutions in France or abroad, or from public or private research centers.
L'archive ouverte pluridisciplinaire HAL, est destinée au dépôt et à la diffusion de documents scientifiques de niveau recherche, publiés ou non, émanant des établissements d'enseignement et de recherche français ou étrangers, des laboratoires publics ou privés. 


\title{
Material Decomposition in X-ray Spectral CT Using Multiple Constraints in Image Domain
}

\author{
Bingqing Xie ${ }^{1}$, Ting $\mathrm{Su}^{2}$, Valérie Kaftandjian ${ }^{2}$, Pei Niu${ }^{1}$, Feng Yang ${ }^{3}$, Marc Robini ${ }^{1}$, Yuemin $\mathrm{Zhu}^{1}$, Philippe Duvauchelle ${ }^{2}$ \\ ${ }^{1}$ Univ Lyon, INSA Lyon, CNRS, Inserm, CREATIS UMR 5220, U1206, F-69621, Lyon, France, e-mail: \\ yue-min.zhu@creatis.insa-lyon.fr \\ ${ }^{2}$ Univ Lyon, INSA Lyon, Laboratoire Vibrations Acoustique, F-69621 Villeurbanne, France \\ ${ }^{3}$ Beijing Jiaotong Univ, School of Computer and Information Technology, Beijing, China
}

\begin{abstract}
X-ray spectral CT appears as a new promising imaging modality for the quantitative measurement of materials in an object, compared to conventional energy-integrating CT or dual energy CT. We consider material decomposition in spectral CT as an overcomplete ill-conditioned inverse problem. To solve the problem, we make full use of multi-dimensional nature and high correlation of multi-energy data and spatially neighboring pixels in spectral CT. Meanwhile, we also exploit the fact that material mass density has limited value. The material decomposition is then achieved by using bounded mass density, local joint sparsity and structural low-rank (DSR) in image domain. The results on numerical phantom demonstrate that the proposed DSR method leads to more accurate decomposition than usual pseudo-inverse method with singular value decomposition (SVD) and current popular sparse regularization method with $\ell 1$-norm constraint.
\end{abstract}

\section{Keywords: X-ray spectral CT, material decomposition, sparse representation, low-rank representation}

\section{Introduction}

Multi-energy CT or spectral CT has recently received increasingly intensive attention, due to the new advances in photon counting detector technology. Compared to conventional energy integrating CT or dual-energy CT, spectral CT can count the number of photons in separated energy bins with one single exposure [1]. Thanks to this advantage, it becomes possible to decompose efficiently different materials (basis materials) present in the same pixel of spectral CT images, which provides promising perspectives for both medical applications and industrial nondestructive evaluation.

According to the type and number of basis materials, material decomposition in spectral CT can be an ill-posed, or even illconditioned inverse problem. Such problem can arise before [2-4], during [5], or after [6] image reconstruction process. A number of material decomposition methods were reported in the literature. They can be categorized into two main types: optimization without and with regularization. The pseudo-inverse method with singular value decomposition (SVD) is a usual optimization method without regularization [6]. In the optimization method with regularization, sparse representation was often employed as that multi-energy data often has high correlation. The major motivation of sparse representation is to make use of the sparsity nature of multi-dimensional data. An appropriate sparse representation can give an accurate material decomposition with few energy bins. Typically, sparse representation via $\ell 1$-norm minimization is considered efficient in many areas [7,8] to prevent oversmoothing. Other methods based on sparse representation were also studied for material decomposition, including gradient sparsity via bilateral total variation (BTV) [9] and sparse transform via tight frame [10]. All the above-cited methods were developed in overdetermined cases; no work is available for material decomposition in underdetermined cases where the number of basis materials is greater than that of energy bins.

Actually, in practice an object always contains multiple materials and we do not know a priori what exactly the number of materials in it is. Therefore, material decomposition in underdetermined cases remains a practical problem. The fact that basis materials with close atomic numbers have similar mass attenuation coefficients comes to further worsen the problem. To cope with these problems, we propose to use the joint sparsity [11] characteristics of both spectrum and space information. Moreover, since neighboring pixels have similar materials, we exploit the joint sparsity in local small patches. Meanwhile, we also make use of the structural low-rank property that the decomposed materials in the corresponding local small patch should share the same edge, due to the local property that, in a local patch, a decomposed material is either present or absent at each pixel of the patch. Finally, since the mass density of materials has always limited value, we introduce a bound constraint on mass density. All that leads us to propose a material decomposition method based on bounded mass Density, local joint Sparsity and structural low-Rank (DSR).

\section{Model and Method}

\subsection{Model of material decomposition in image domain}

For CT images, the object model of material decomposition is described by: 
$\mu(\vec{x}, E)=\sum_{\alpha=1}^{M} \rho_{\alpha}(\vec{x}) \mu_{m \alpha}(E)$,

where $\mu(\vec{x}, E)$ represents the linear attenuation coefficient, $\vec{x}$ the position or pixel and $E$ the energy. $\mu(\vec{x}, E)$ also denotes the reconstructed value from acquisitions. It is considered a linear combination of the mass attenuation coefficients $\mu_{m \alpha}(E)$ of $M$ basis materials weighted by the corresponding mass density $\rho_{\alpha}(\vec{x})$ at pixel $\vec{x}$.

The aim of material decomposition is to solve $\rho_{\alpha}(\vec{x})$ from the above equation. For spectral CT, we can rewrite the object model into its matrix form:

$$
\underbrace{\left[\begin{array}{ccc}
\mu\left(\vec{x}_{1}, 1\right) & \ldots & \mu\left(\vec{x}_{N}, 1\right) \\
\vdots & \ddots & \vdots \\
\mu\left(\vec{x}_{1}, B\right) & \cdots & \mu\left(\vec{x}_{N}, B\right)
\end{array}\right]}_{\boldsymbol{Y}}=\underbrace{\left[\begin{array}{ccc}
\mu_{m 1}(1) & \ldots & \mu_{m M}(1) \\
\vdots & \ddots & \vdots \\
\mu_{m 1}(B) & \cdots & \mu_{m M}(B)
\end{array}\right]}_{\boldsymbol{A}} \cdot \underbrace{\left[\begin{array}{ccc}
\rho_{1}\left(\vec{x}_{1}\right) & \ldots & \rho_{1}\left(\vec{x}_{N}\right) \\
\vdots & \ddots & \vdots \\
\rho_{M}\left(\vec{x}_{1}\right) & \cdots & \rho_{M}\left(\vec{x}_{N}\right)
\end{array}\right]}_{\boldsymbol{X}},
$$

where $B$ is the number of energy bins, $N$ the number of pixels in one patch that represents a small square region of the image, and $\mu\left(\vec{x}_{N}, B\right)$ the value of linear attenuation coefficient of the $B$-th energy bin at the $N$-th pixel. The capital letters $\boldsymbol{Y}, \boldsymbol{A}$ and $\boldsymbol{X}$ in equation (2) represent the matrix form of the object model. Each column of $\boldsymbol{A}$ represents the mass attenuation coefficients of a given material for the $B$ energies, and each column of $\boldsymbol{Y}$ represents the energy-dependent linear attenuation coefficients at a pixel. In practical applications, we do not know a priori about the materials that constitute an object and therefore we need to take a larger number of material types into consideration, thus leading to underdetermined linear inverse problems $(B<M)$.

\subsection{Material decomposition using multiple constraints}

For spectral CT images, basis materials with close atomic numbers have similar mass attenuation coefficients, and this is the source of ill-conditioned problems and constitutes the main bottleneck that limits the performance of material decomposition methods. To cope with this problem, we propose to find the main features by exploiting the similarities in multi-dimensional data.

To this end, we use sparse representation allowing processing the high correlation of both spectral and spatial dimensions. Meanwhile, the non-zero rows (actually existing materials) in $\boldsymbol{X}$ of equation (2) are also sparse compared to the great number of basis materials in $\boldsymbol{X}$. Such joint sparsity of spectral and spatial dimensions would be interesting for decomposing similar materials in a robust way. We then adopt the mixed $\ell_{2,1}$-norm that is particularly suitable for small patches to combine the information. The norm $\ell_{2,1}$ is defined by:

$\|\boldsymbol{X}\|_{2,1}=\sum_{i=1}^{I} \sqrt{\sum_{j=1}^{J} x_{i j}^{2}}$,

where $x_{i j}$ is the mass density of the $i$-th basis material at the $j$-th pixel in $\boldsymbol{X}$ of equation (2). $\ell_{2,1}$-norm can enforce a small number of nonzero rows in $\boldsymbol{X}$, compared to usual $\ell_{1}$-norm $\|\boldsymbol{X}\|_{1}=\sum_{i=1}^{I} \sum_{j=1}^{J}\left|x_{i j}\right|$ that tends to give sparse solution pixel by pixel. In other words, $\ell_{1}$-norm limits the number of basis materials exiting in one pixel, while $\ell_{2,1}$-norm enforces pixels to share the same atoms of basis materials in each local small patch. For the present application of spectral CT images, the $\ell_{2,1}$-norm allows us to combine space (rows in $\boldsymbol{X}$ ) and spectrum (columns in $\boldsymbol{X}$ ) information.

At the same time, in addition to the spectral and spatial similarities, multi-energy images also present the structural coherence, which reflects the fact that the decomposed materials should share the same edges in the same small patch due to their local property in the image. Such edge information in a material image is also sparse and can be captured by gradient operator. To process the edge similarities, we utilize low-rank representation on the gradient matrices of all the decomposed materials. The low-rank constraint is realized via nuclear norm that is the convex envelope of matrix rank [12]. As a result, the structural lowrank (SL) is calculated using:

$\|\boldsymbol{\nabla} \boldsymbol{X}\|_{* *}=\sum_{i=1} \sigma_{i}(\boldsymbol{\nabla} \boldsymbol{X})$,

where $\boldsymbol{\nabla} \boldsymbol{X}$ is the gradient matrix of $\boldsymbol{X}$ and $\|\boldsymbol{\nabla} \boldsymbol{X}\|_{*}$ is the nuclear norm equal to the sum of its singular values $\sigma_{i}(\boldsymbol{\nabla} \boldsymbol{X})$. Actually, this kind of rank-sparsity encouraging norm has also been discussed in color image denoising, with the name total nuclear variation (TNV) [13]. However, in the present study, it is utilized to process the structural similarity that existing material images $\boldsymbol{X}_{\boldsymbol{i}}$ (nonzero rows in $\boldsymbol{X}$ of equation (2)) should share the same edges.

A cost function containing the joint sparsity and low-rank regularizations and a data-fidelity term may give an optimal solution to the problem of material decomposition. We then formulate our material decomposition method based on local joint sparsity and structural low-rank as:

$\underset{X}{\arg \min } \frac{1}{2}\left\|\boldsymbol{Y}_{\boldsymbol{P}}-\boldsymbol{A} \boldsymbol{X}_{P}\right\|_{F}^{2}+\lambda_{1}\left\|\boldsymbol{X}_{\boldsymbol{P}}\right\|_{2,1}+\lambda_{2}\left\|\boldsymbol{\nabla} \boldsymbol{X}_{\boldsymbol{P}}\right\|_{*}$, 
where $Y_{\boldsymbol{P}}$ and $\boldsymbol{X}_{\boldsymbol{p}}$ denote the patches extracted respectively from the reconstructed images and the decomposed material images and $\lambda_{1}, \lambda_{2}>0$ are the scalar regularization parameters.

At the same time, since the mass density of materials always has limited value, we introduce the bound constraints into equation (5). In other words, the mass densities of materials should be lower bounded by a nonnegative number and upper bounded by a reasonable limit. Thus, after taking into account the multiple constraints of bounded mass Density, local joint Sparsity and structural low-Rank (DSR), our material decomposition model becomes:

$\underset{\boldsymbol{X}}{\arg \min } \frac{1}{2}\left\|\boldsymbol{Y}_{\boldsymbol{P}}-\boldsymbol{A} \boldsymbol{X}_{\boldsymbol{P}}\right\|_{F}^{2}+\lambda_{1}\left\|\boldsymbol{X}_{\boldsymbol{P}}\right\|_{2,1}+\lambda_{2}\left\|\boldsymbol{\nabla} \boldsymbol{X}_{\boldsymbol{P}}\right\|_{*}+\operatorname{bound}_{l}\left(\boldsymbol{X}_{\boldsymbol{P}}\right)+\operatorname{bound}_{u}\left(\boldsymbol{X}_{\boldsymbol{P}}\right)$,

where bound $l\left(\boldsymbol{X}_{\boldsymbol{p}}\right)$ and bound $_{u}\left(\boldsymbol{X}_{\boldsymbol{p}}\right)$ denote the lower and upper mass density bounds, respectively.

\subsection{DSR: solution algorithm by ADMM}

The minimization problem of (6) is solved by the alternating direction method of multipliers (ADMM) [14]. Firstly, we form the augmented Lagrangian for problem (6):

$$
\begin{aligned}
\boldsymbol{L}_{\lambda}\left(\boldsymbol{V}_{1}, \boldsymbol{V}_{2}, \boldsymbol{V}_{3}, \boldsymbol{V}_{4}, \boldsymbol{X}\right)= & \underset{\boldsymbol{V}_{1}, \boldsymbol{V}_{2}, \boldsymbol{V}_{3}, \boldsymbol{V}_{4}, \boldsymbol{X}}{\arg \min } \frac{1}{2}\left\|\boldsymbol{Y}_{\boldsymbol{P}}-\boldsymbol{V}_{1}\right\|_{F}^{2}+\lambda_{1}\left\|\boldsymbol{V}_{2}\right\|_{2,1}+\lambda_{2}\left\|\boldsymbol{V}_{3}\right\|_{*}+\operatorname{bound}_{l}\left(\boldsymbol{V}_{4}\right)+\operatorname{bound}_{u}\left(\boldsymbol{V}_{5}\right) \\
& +\frac{\tau}{2}\left\|\boldsymbol{A} \boldsymbol{X}_{\boldsymbol{P}}-\boldsymbol{V}_{1}-\boldsymbol{D}_{1}\right\|_{F}^{2}+\frac{\tau}{2}\left\|\boldsymbol{X}_{\boldsymbol{P}}-\boldsymbol{V}_{2}-\boldsymbol{D}_{2}\right\|_{F}^{2} \\
& +\frac{\tau}{2}\left\|\boldsymbol{\nabla} \boldsymbol{X}_{\boldsymbol{P}}-\boldsymbol{V}_{3}-\boldsymbol{D}_{3}\right\|_{F}^{2}+\frac{\tau}{2}\left\|\boldsymbol{X}_{\boldsymbol{P}}-\boldsymbol{V}_{4}-\boldsymbol{D}_{4}\right\|_{F}^{2}+\frac{\tau}{2}\left\|\boldsymbol{X}_{\boldsymbol{P}}-\boldsymbol{V}_{5}-\boldsymbol{D}_{5}\right\|_{F}^{2}
\end{aligned}
$$

where $\tau>0$ is the augmented Lagrangian parameter.

ADMM consists of the following iterations:

$$
\begin{aligned}
& \boldsymbol{X}_{\boldsymbol{P}}{ }^{t+1} \leftarrow \underset{\boldsymbol{X}_{\boldsymbol{P}}}{\arg \min } \frac{\tau}{2}\left\|\boldsymbol{A} \boldsymbol{X}_{\boldsymbol{P}}-\boldsymbol{V}_{1}^{t}-\boldsymbol{D}_{1}^{t}\right\|_{F}^{2}+\frac{\tau}{2}\left\|\boldsymbol{X}_{\boldsymbol{P}}-\boldsymbol{V}_{2}^{t}-\boldsymbol{D}_{2}^{t}\right\|_{F}^{2}+\frac{\tau}{2}\left\|\nabla \boldsymbol{X}_{\boldsymbol{P}}-\boldsymbol{V}_{3}-\boldsymbol{D}_{3}\right\|_{F}^{2} \\
& +\frac{\tau}{2}\left\|\boldsymbol{X}_{\boldsymbol{P}}-\boldsymbol{V}_{4}^{t}-\boldsymbol{D}_{4}^{t}\right\|_{F}^{2}+\frac{\tau}{2}\left\|\boldsymbol{X}_{\boldsymbol{P}}-\boldsymbol{V}_{5}^{t}-\boldsymbol{D}_{5}^{t}\right\|_{F}^{2} \\
& \boldsymbol{V}_{1}^{t+1} \leftarrow \underset{V_{1}}{\arg \min } \frac{1}{2}\left\|\boldsymbol{Y}_{\boldsymbol{P}}-\boldsymbol{V}_{1}\right\|_{F}^{2}+\frac{\tau}{2}\left\|\boldsymbol{A} \boldsymbol{X}_{\boldsymbol{P}}{ }^{t}-\boldsymbol{V}_{1}-\boldsymbol{D}_{1}{ }^{t}\right\|_{F}^{2}, \\
& \boldsymbol{V}_{2}^{t+1} \leftarrow \underset{V_{2}}{\arg \min } \lambda_{1}\left\|\boldsymbol{V}_{2}\right\|_{2,1}+\frac{\tau}{2}\left\|\boldsymbol{X}_{\boldsymbol{P}}{ }^{t}-\boldsymbol{V}_{2}-\boldsymbol{D}_{2}{ }^{t}\right\|_{F}^{2}, \\
& \boldsymbol{V}_{3}^{t+1} \leftarrow \underset{V_{3}}{\arg \min } \lambda_{2}\left\|\boldsymbol{V}_{3}\right\|_{*}+\frac{\tau}{2}\left\|\nabla \boldsymbol{X}_{\boldsymbol{P}}-\boldsymbol{V}_{3}-\boldsymbol{D}_{3}\right\|_{F}^{2}, \\
& \boldsymbol{V}_{4}{ }^{\boldsymbol{t}+1} \leftarrow \underset{\boldsymbol{V}_{4}}{\arg \min } \text { bound }_{l}\left(\boldsymbol{V}_{4}\right)+\frac{\tau}{2}\left\|\boldsymbol{X}_{\boldsymbol{P}}{ }^{\boldsymbol{t}}-\boldsymbol{V}_{4}-\boldsymbol{D}_{4}{ }^{t}\right\|_{F}^{2}, \\
& \boldsymbol{V}_{5}^{t+1} \leftarrow \underset{V_{5}}{\arg \min } \operatorname{bound}_{u}\left(\boldsymbol{V}_{5}\right)+\frac{\tau}{2}\left\|\boldsymbol{X}_{\boldsymbol{P}}{ }^{\boldsymbol{t}}-\boldsymbol{V}_{5}-\boldsymbol{D}_{5}{ }^{t}\right\|_{F}^{2} .
\end{aligned}
$$

Equations (8) to (13) aim to solve primal variables $\boldsymbol{X}_{\boldsymbol{P}}, \boldsymbol{V}_{1}, \boldsymbol{V}_{2}, \boldsymbol{V}_{3}, \boldsymbol{V}_{4}$ and $\boldsymbol{V}_{5}$. Equation (10) is processed by the algorithm FOCUSS [15, 16], and equation (11) by the singular value thresholding (SVT) method [17]. The solutions of $\boldsymbol{X}_{\boldsymbol{P}}, \boldsymbol{V}_{1}, \boldsymbol{V}_{2}, \boldsymbol{V}_{3}, \boldsymbol{V}_{4}$ and $\boldsymbol{V}_{5}$ are given by:

$$
\begin{aligned}
& \boldsymbol{X}_{\boldsymbol{P}}{ }^{t+1}=\left(\boldsymbol{A}^{\boldsymbol{T}} \boldsymbol{A}+\boldsymbol{\nabla}^{\boldsymbol{T}} \boldsymbol{\nabla}+3 \boldsymbol{I}\right)^{-1}\left[\boldsymbol{A}^{\boldsymbol{T}}\left(\boldsymbol{V}_{1}^{\boldsymbol{t}}+\boldsymbol{D}_{1}^{\boldsymbol{t}}\right)+\left(\boldsymbol{V}_{2}^{t}+\boldsymbol{D}_{2}^{t}\right)\right. \\
& \left.+\nabla^{T}\left(V_{3}^{t}+D_{3}^{t}\right)+\left(V_{4}^{t}+D_{4}^{t}\right)+\left(u b-V_{5}^{t}-D_{5}^{t}\right)\right], \\
& \boldsymbol{V}_{1}^{t+1}=\frac{1}{1+\tau}\left[\boldsymbol{Y}_{\boldsymbol{P}}+\tau\left(\boldsymbol{A} \boldsymbol{X}_{P}{ }^{t}-\boldsymbol{D}_{1}^{t}\right)\right] \\
& \boldsymbol{V}_{2}{ }^{t+1}=\left(\boldsymbol{X}_{\boldsymbol{P}}{ }^{t}-\boldsymbol{D}_{2}{ }^{t}\right) \cdot\left(\boldsymbol{I}-\frac{\lambda_{1} / \tau}{\left\|\boldsymbol{X}_{\boldsymbol{P}}{ }^{t}-\boldsymbol{D}_{2}{ }^{t}\right\|_{2}}\right)^{+}, \\
& \boldsymbol{V}_{3}^{t+1}=\operatorname{SVT}\left(\nabla X_{P}-D_{3}, \frac{\lambda_{2}}{\tau}\right), \\
& \boldsymbol{V}_{4}^{t+1}=\left(\boldsymbol{X}_{\boldsymbol{P}}{ }^{t}-\boldsymbol{D}_{4}{ }^{t}\right)^{+}, \\
& \boldsymbol{V}_{5}^{t+1}=\left(\boldsymbol{u b}-\boldsymbol{X}_{\boldsymbol{P}}{ }^{\boldsymbol{t}}-\boldsymbol{D}_{5}{ }^{\boldsymbol{t}}\right)^{+},
\end{aligned}
$$

where $\boldsymbol{u} \boldsymbol{b}$ is the upper bound vector and $(\boldsymbol{x})^{+}=\max (\boldsymbol{x}, 0)$.

For clarity, the detail of ADMM for the DSR decomposition method is given in Algorithm 1. The iteration will stop when the criterion of ADMM is satisfied, e.g. the residuals are small enough. 
Algorithm 1: ADMM for the DSR decomposition method

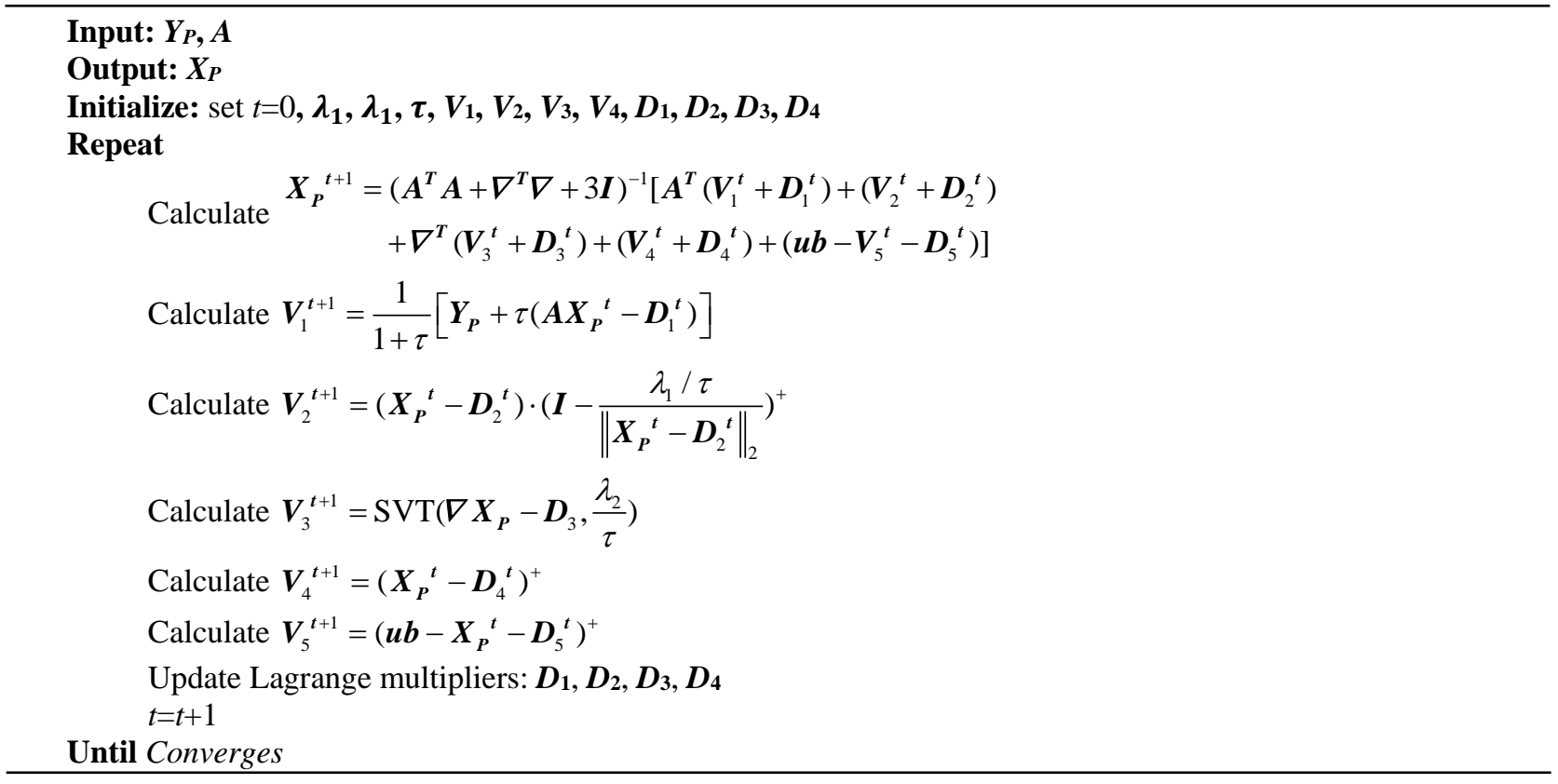

\section{Results}

\subsection{Phantom}

The projection data of spectral CT was simulated using INSA software Virtual X-ray Imaging (VXI) [18] and then reconstructed by the method Filtered Backprojection (FBP). The reconstructed phantom has $800 \times 800$ pixels and contains five materials: water, Polymethyl Methacrylate (PMMA), gadolinium $(G d)$, iodine $(I)$ and iron $(F e)$, as shown in Figure 1 . The number on each disk designates the concentration of materials $(\mathrm{mg} / \mathrm{cc})$. Note that the disk with \# stands for mixture inserts that contain three basis materials $(G d, I$ and $F e$ ) with the same concentration in each column. The X-ray energy bins were set as: 20 30kev, 30 40kev, 40 50kev, 50 60kev, 60 70kev and 70 80kev.

To evaluate the performance of the proposed DSR method, we considered 20 materials as basis materials divided into 4 categories, including the five materials (bold ones) present in the phantom, as listed in Table 1. Because most elements in the human body have relatively small atomic numbers, we put low-Z elements: sodium $(\mathrm{Na})$, magnesium $(\mathrm{Mg})$, aluminum $(\mathrm{Al})$, silicon $(\mathrm{Si})$, phosphorus $(P)$, sulfur $(S)$, chlorine $(C l)$, argon $(A r)$ and potassium $(K)$ into the basis materials as category 1 . Manganese $(\mathrm{Mn})$, cobalt $(\mathrm{Co})$, copper $(\mathrm{Cu})$ and zinc $(\mathrm{Zn})$ were selected for comparison with $\mathrm{Fe}$, as category 2 . To test the accuracy of $I$ and $G d$, another common contrast agent xenon $(\mathrm{Xe})$ was also added, as category 3 . Bone was selected to test the accuracy of water and PMMA because of their similar attenuation coefficients. The mass attenuation coefficients were retrieved from NIST [19].

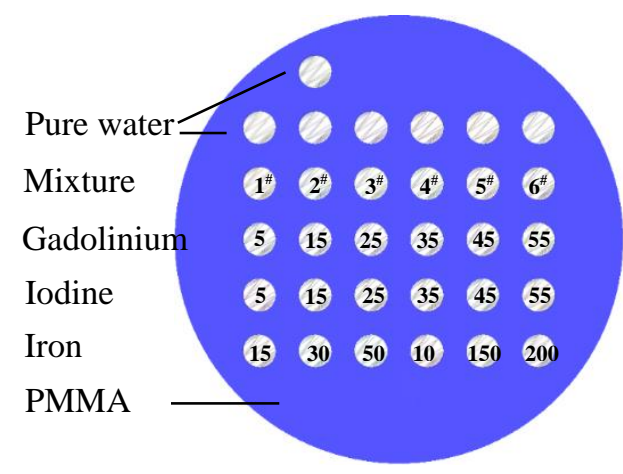

Figure 1: Digital phantom.
Table 1: Basis materials and the corresponding atomic numbers.

\begin{tabular}{|c|c|c|c|c|c|c|c|c|c|c|}
\hline \multirow{2}{*}{ Category 1} & $\mathrm{M}$ & $\mathrm{Na}$ & $M g$ & $A l$ & $S i$ & $P$ & $S$ & $\mathrm{Cl}$ & $A r$ & $K$ \\
\hline & $\mathrm{Z}$ & 11 & 12 & 13 & 14 & 15 & 16 & 17 & 18 & 19 \\
\hline \multirow{2}{*}{ Category 2} & $\mathrm{M}$ & $M n$ & $F e$ & $\mathrm{Co}$ & $\mathrm{Cu}$ & $\mathrm{Zn}$ & & & & \\
\hline & $\mathrm{Z}$ & 25 & 26 & 27 & 29 & 30 & & & & \\
\hline \multirow{2}{*}{ Category 3} & $\mathrm{M}$ & $I$ & $X e$ & $G d$ & & & & & & \\
\hline & $\mathrm{Z}$ & 53 & 54 & 64 & & & & & & \\
\hline \multirow{2}{*}{ Category 4} & $\mathrm{M}$ & water & PMMA & bone & & & & & & \\
\hline & $\mathrm{Z}$ & & mixture & & & & & & & \\
\hline
\end{tabular}




\subsection{DSR versus other methods}

Two methods were compared with the proposed DSR method: (a) pseudo-inverse with SVD, (b) regularization method with $\ell 1$ norm constraint ( $\ell 1)$. For clarity, we rewrite the models of the three methods as below:

(a) Pseudo-inverse with SVD: $\boldsymbol{X}=\boldsymbol{A}^{-1} \boldsymbol{Y}$,

(b) $\ell 1: \underset{\boldsymbol{X}}{\arg \min } \frac{1}{2}\|\boldsymbol{Y}-\boldsymbol{A} \boldsymbol{X}\|_{F}^{2}+\lambda\|\boldsymbol{X}\|_{1}$,

(c) DSR: $\underset{\boldsymbol{X}}{\arg \min } \frac{1}{2}\left\|\boldsymbol{Y}_{\boldsymbol{P}}-\boldsymbol{A} \boldsymbol{X}_{\boldsymbol{P}}\right\|_{F}^{2}+\lambda_{1}\left\|\boldsymbol{X}_{\boldsymbol{P}}\right\|_{2,1}+\lambda_{2}\left\|\boldsymbol{\nabla} \boldsymbol{X}_{\boldsymbol{P}}\right\|_{*}+\operatorname{bound}_{l}\left(\boldsymbol{X}_{\boldsymbol{P}}\right)+\operatorname{bound}_{u}\left(\boldsymbol{X}_{\boldsymbol{P}}\right)$.

The decomposition results of five existing basis materials are shown in Figure 2. Note that in the present study, the lower mass density bound is set as 0 and the upper bound as 10 times of the maximum mass density value of existing materials. Moreover, only nonnegative decomposition results were kept, considering that negative mass density is meaningless in practical applications The method pseudo-inverse with SVD can neither quantify the 5 existing basis materials nor the remaining 13 basis materials (not shown in Figure 2 due to limited space). It can be partly explained by the condition number $\kappa(A)$ of matrix, which is equal to the ratio of the largest to smallest singular value, and is used to measure how sensitive a function is to errors in the input [20]. A problem with a high condition number is ill-conditioned. In our experiments, there are 20 similar basis materials, leading to a big condition number $\kappa(A)=1.7 \times 10^{5}$, which implies that the result of usual pseudo-inverse with SVD is not reliable for our ill-condition problem. Therefore, we will focus on the other two methods. Note that the images in each column have the same index of color bar.
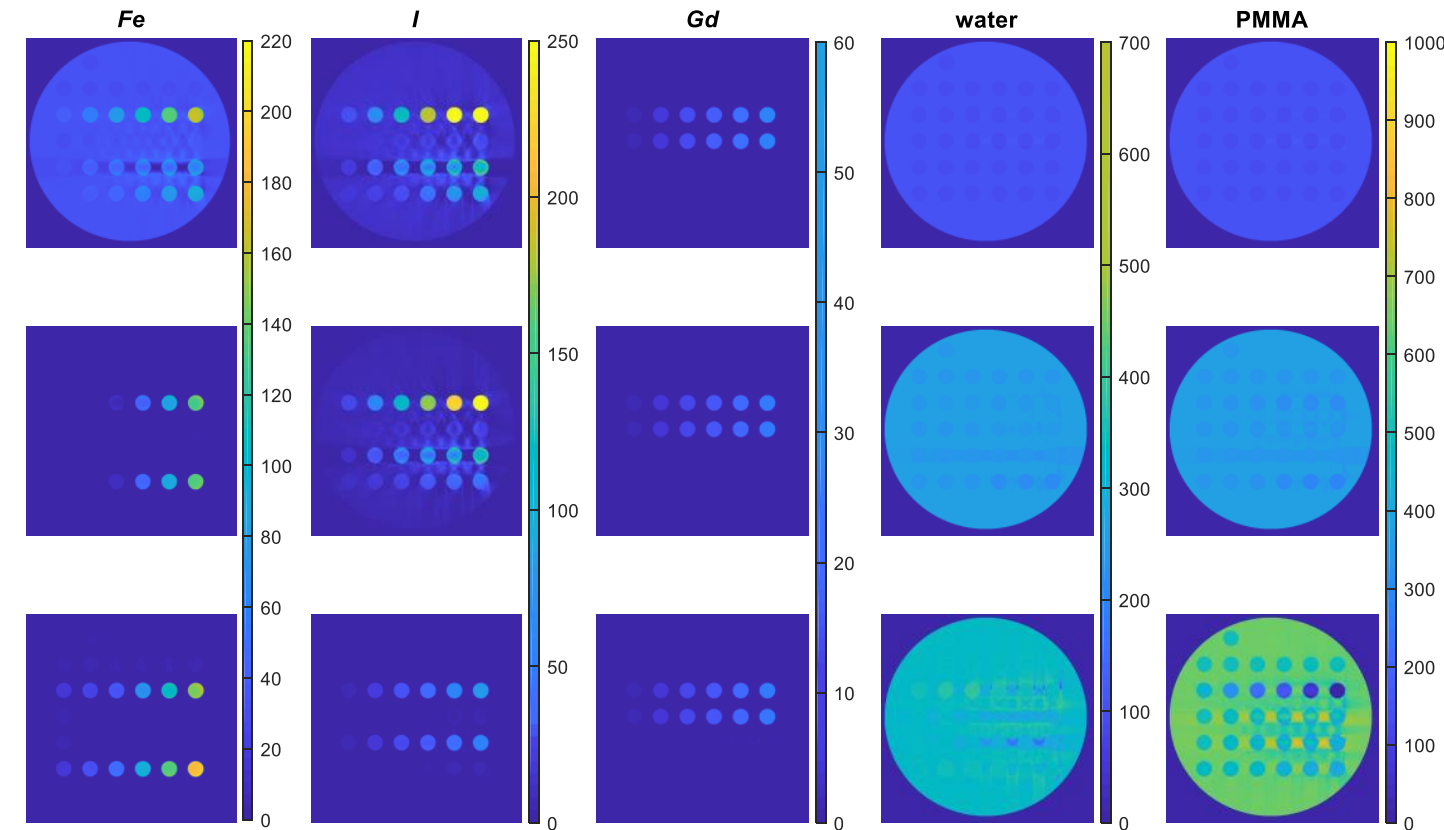

Figure 2: The results of material decomposition using the three methods for five existing basis materials.

Top to bottom: Pseudo-inverse with SVD, $\ell 1$ and DSR.

As can be seen in Figure 2, the proposed DSR method has higher decomposition accuracy compared to the $\ell_{1}$ method. Even for the material without k-edge $(F e)$, DSR shows good ability in detection and quantification. For more quantitative analysis, we compare the mass density of $F e, I$ and $G d$ in the corresponding rows. The results indicate that DSR has good decomposition ability for all of iron, iodine and gadolinium, as shown in Figure 3. In contrast, $\ell 1$ yields much lower density of iron and higher density of iodine compared to the theoretical values.

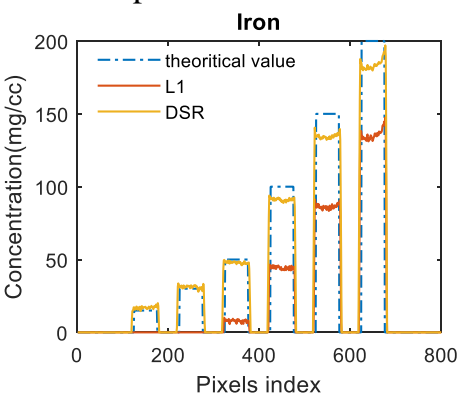

(a)

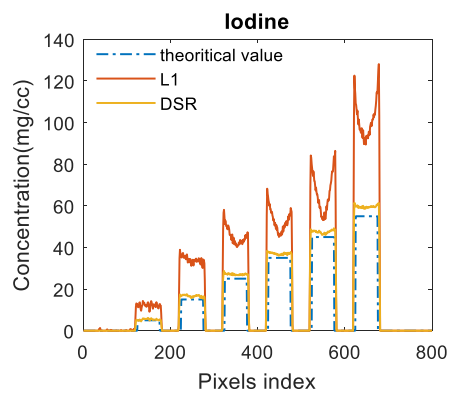

(b)

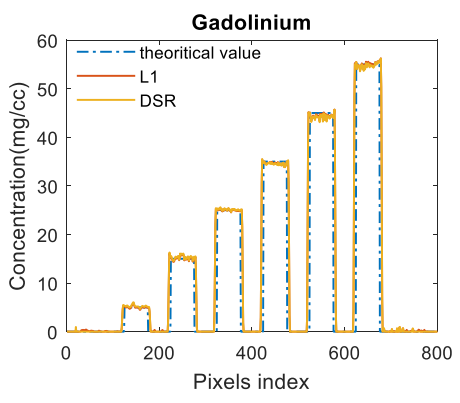

(c)

Figure 3: The mass densities of iron, iodine and gadolinium 
To make a comprehensive evaluation of the proposed DSR, the results of material decomposition for the remaining categories of basis materials in Table 1 are given in the following. The decomposition results of materials in the category 1 are shown in Figure 4, in which we show the results only for the elements $\mathrm{Na}, \mathrm{Mg}, \mathrm{Al}$ and $\mathrm{Si}$ since the decomposed images have zero value

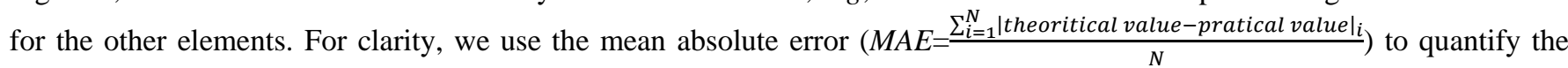
accuracy. The $M A E$ s of basis materials in the category 1 are plotted in the logarithmic coordinates, as shown in Figure 5. For $\ell 1$, although the $M A E$ s of the elements $P, S, C l, A r$ and $K$ are zero, the $M A E$ s of other elements $N a, M g, A l$ and $S i$ are not negligible. Compared with those of $\ell 1$, the MAEs of DSR are much smaller, which implies that the proposed method has better ability to distinguish existing materials from the low-Z materials in the category 1 .

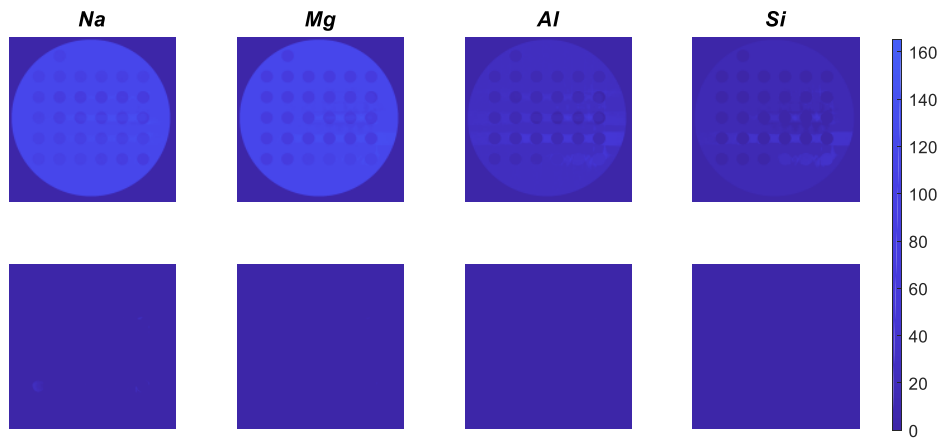

Figure 4: The results of material decomposition using the two methods for the basis materials in the category 1. Top to bottom: $\ell 1$ and DSR.



Figure 5: The MAEs of materials in the category 1 .

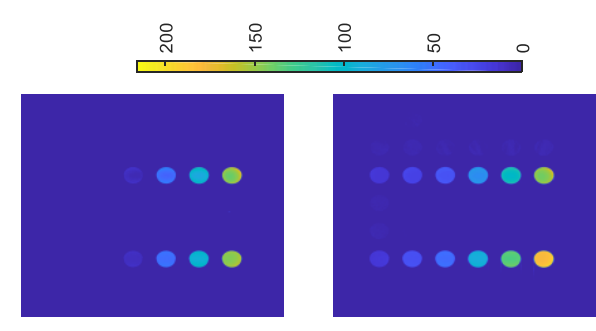

Figure 6: The results of material decomposition using the two methods for the basis material $F e$ in the category 2. Left to right: $\ell 1$ and DSR.

To evaluate the detection ability for iron, the decomposition results of basis materials in the category 2 are shown in Figure 6 where we show only the basis material $\mathrm{Fe}$ since the decomposed images corresponding to the basis materials $\mathrm{Mn}$, $\mathrm{Co}, \mathrm{Cu}$ and $Z n$ have zero value. Both of $\ell 1$ and DSR have good performance to distinguish $F e$ from similar materials $M n, C o, C u$ and $Z n$. However, $\ell 1$ does not have a good quantitative performance for $F e$. Actually, $\ell 1$ cannot detect $F e$ having a concentration below $30 \mathrm{mg} / \mathrm{cc}$ in our experiments, as also shown in Figure 3(a). In contrast, DSR has better ability in detecting and quantifying $F e$ from similar basis materials.

To evaluate the decomposition performance of the proposed method in the presence of contrast agent, the decomposition results of basis materials in the category 3 are given in Figure 7. The proposed DSR presents smaller errors than $\ell 1$ for the contrast agents $I, X e$ and $G d$. Contrast agents have wide applications in the field of medical and industrial imaging, and a good decomposition ability of this kind of materials is important.
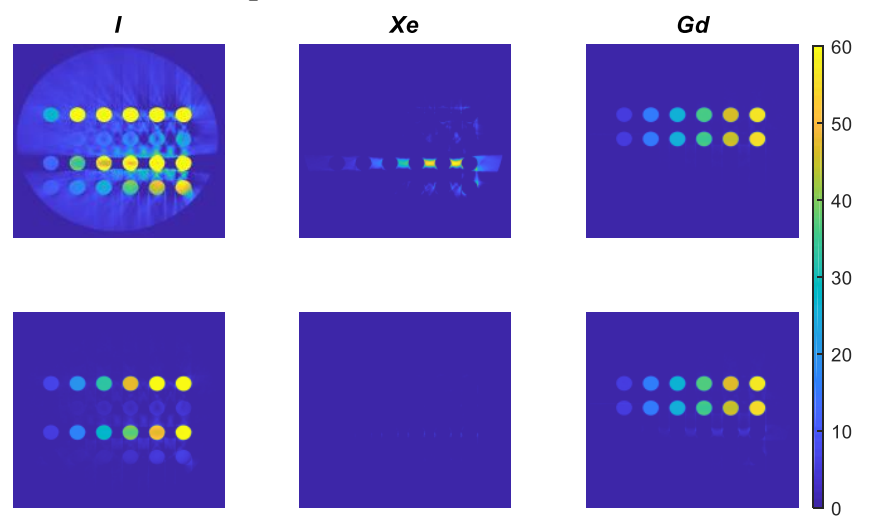

Figure 7: The results of material decomposition using the two methods for basis materials in the category 3.

Top to bottom: $\ell 1$ and DSR. 
For mixture, both of the methods $\ell 1$ and DSR cannot separate water and PMMA, as shown in Figure 8. However, DSR can distinguish bone from water and PMMA. That might be because water and PMMA have closer attenuation coefficients compared to the attenuation coefficient of bone, as shown in Figure 9. This similar attenuation can be quantified by Euclidean distance between two multi-dimensional vectors. The Euclidean distance of two vectors $\left[x_{11}, x_{12}, \ldots, x_{1 n}\right]$ and $\left[x_{21}, x_{22}, \ldots, x_{2 n}\right]$ is defined by:

$d_{12}=\sqrt{\sum_{k=1}^{n}\left(x_{1 k}-x_{2 k}\right)^{2}}$,

where $n$ is the dimension of vector. The Euclidean distance between water and PMMA $d_{\text {water-PMMA }}$ is 0.12 , while for water and bone, $d_{\text {water-bone }}$ equals 1.33 . Normally, two elements with similar atomic numbers have close attenuation coefficients and their Euclidean distance is relatively small. Furthermore, we analysed the Euclidean distances between $F e$ and the basis materials in the category 1. As shown in Figure 10, when the atomic number of materials in the category 1 increases to that of iron, the Euclidean distance decreases gradually.
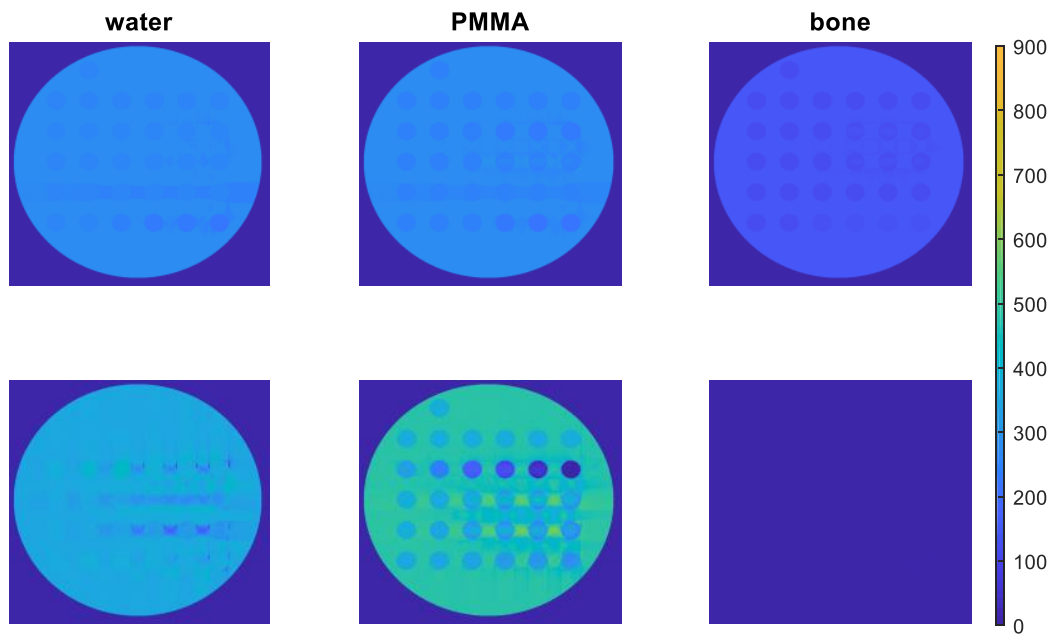

Figure 8: The results of material decomposition using the two methods for basis materials in the category 3 . Top to bottom: $\ell 1$ and DSR.

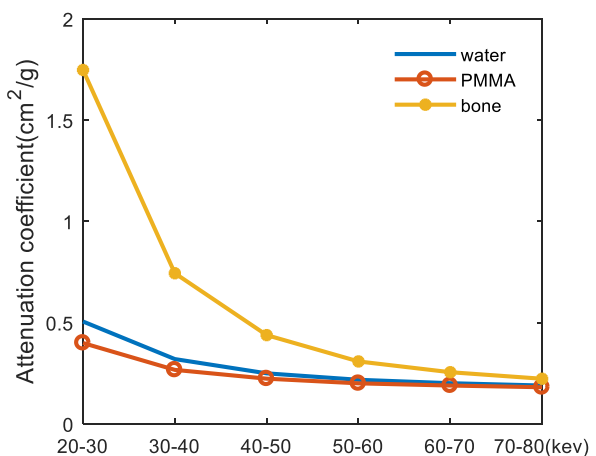

Figure 9: Equivalent attenuation coefficients of water, PMMA and bone in the 6 energy bins.

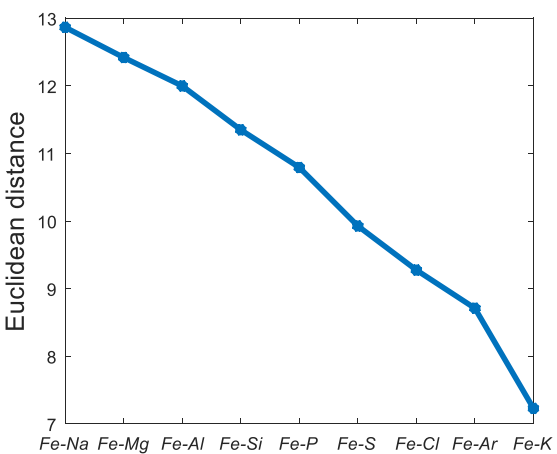

Figure 10: Euclidean distance between iron and basis materials in the category 1 .

\section{Discussion and conclusion}

We have proposed a material decomposition method for spectral CT by simultaneously exploiting the limited value of mass density, the multi-dimensional nature and high correlation of multi-energy data and the local property of neighboring pixels. To get rid of the difficulties due to similarities among basis materials, spectral and spatial redundancies are eliminated through using joint sparsity and structural low-rank. At the same time, we also add bound constraint on mass density, which is naturally true in practical applications. Preliminary results revealed that the proposed DSR method has a good material decomposition ability even for very poorly conditioned problems.

The regularization in the proposed DSR method is a convex problem. We have used an iterative algorithm ADMM to solve the proposed model. To accelerate convergence, the initialization of variables in the ADMM was set as the results of pseudoinverse with SVD. Although there are four regularizations in the cost function, only the joint sparsity term and the structural low-rank term have the regularization parameters $\lambda_{1}$ and $\lambda_{2}$. The results of material decomposition are clearly influenced by the regularization parameters. This is in fact a persistent problem, since solving cost function with more than one regularization 
parameter is still a challenging problem $[9,10,21,22]$. The scalar regularization parameters $\lambda_{1}$ and $\lambda_{2}$ influence respectively the level of sparsity and low-rank. We have chosen the two scalar parameters by cross-validation [14]. The augmented Lagrangian parameter $\tau$ that is equal to the step size of iteration has also an obvious impact on convergence speed. In practice, to make performance less dependent on the initial choice, we have updated $\tau$ in each iteration by the way discussed in [14, 23] (try to keep the primal and dual residual norms in the ADMM within a factor as they both converge to zero). Finally, due to the use of local property, the choice of regularization parameters $\lambda_{1}, \lambda_{2}$ and $\tau$ is also influenced by the size of patch. Generally, a smaller size of patch should have the correspondingly smaller regularization parameters, otherwise obvious errors will occur near the edges of patches in the decomposed material images. Note that neither too large patches nor too small patches accords with the local property or result in an accurate decomposition, and it should be set according to the property of each spectral CT data.

In conclusion, the proposed DSR method exhibits good performance in separating and quantifying many kinds of materials, e.g. elements with small atomic number and contrast agents. In particular, the good ability of DSR to distinguish similar contrast agents (e.g. iodine, gadolinium and xenon) suggests its interesting potential use for industrial and medical applications. In the future work, the collaborative joint sparsity and structural low-rank should be further investigated to make full use of the sparsity and low-rank property hidden in the spectral CT data. Finally, a more robust strategy for the choice of regularization parameters could also be investigated.

\section{References}

1. Faby, S., Kuchenbecker, S., Sawall, S., Simons, D., Schlemmer, H.-P., Lell, M., Kachelrieß, M.: Performance of today's dual energy CT and future multi energy CT in virtual non-contrast imaging and in iodine quantification: A simulation study. Med. Phys. 42, 4349-4366 (2015). doi:10.1118/1.4922654

2. Schlomka, J.P., Roessl, E., Dorscheid, R., Dill, S., Martens, G., Istel, T., Bäumer, C., Herrmann, C., Steadman, R., Zeitler, G., Livne, A., Proksa, R.: Experimental feasibility of multi-energy photon-counting K-edge imaging in preclinical computed tomography. Phys. Med. Biol. 53, 4031-4047 (2008). doi:10.1088/0031-9155/53/15/002

3. Roessl, E., Proksa, R.: K-edge imaging in x-ray computed tomography using multi-bin photon counting detectors. Phys. Med. Biol. 52, 4679-4696 (2007). doi:10.1088/0031-9155/52/15/020

4. Maaß, N., Sawall, S., Knaup, M., Kachelrieß, M.: Empirical multiple energy calibration (EMEC) for material-selective CT. IEEE Nucl. Sci. Symp. Conf. Rec. 4222-4229 (2012). doi:10.1109/NSSMIC.2011.6153810

5. Foygel Barber, R., Sidky, E.Y., Gilat Schmidt, T., Pan, X.: An algorithm for constrained one-step inversion of spectral CT data. Phys. Med. Biol. 61, 3784-3818 (2016). doi:10.1088/0031-9155/61/10/3784

6. $\quad$ Faby, S., Kuchenbecker, S., Simons, D., Schlemmer, H.-P., Lell, M., Kachelrieß, M.: CT calibration and dose minimization in image-based material decomposition with energy-selective detectors. Proc. SPIE Med. Imaging. 9033, 903318 (2014). doi:10.1117/12.2042877

7. Candes, E.J., Romberg, J., Tao, T.: Robust uncertainty principles: exact signal reconstruction from highly incomplete frequency information. IEEE Trans. Inf. Theory. 52, 489-509 (2006). doi:10.1109/TIT.2005.862083

8. Donoho, D.L.: Compressed sensing. IEEE Trans. Inf. Theory. 52, 1289-1306 (2006). doi:10.1109/TIT.2006.871582

9. Clark, D.P., Badea, C.T.: Spectral diffusion: an algorithm for robust material decomposition of spectral CT data. Phys. Med. Biol. 59, 6445-6466 (2014). doi:10.1088/0031-9155/59/21/6445

10. Gao, H., Yu, H., Osher, S., Wang, G.: Multi-energy CT based on a prior rank, intensity and sparsity model (PRISM). Inverse Probl. 27, (2011). doi:10.1088/0266-5611/27/11/115012

11. Nie, F., Huang, H., Cai, X., Ding, C.H.: Efficient and robust feature selection via joint $\ell 2$, 1-norms minimization. In: Advances in Neural Information Processing Systems. pp. 1813-1821 (2010)

12. Can Es, E.J., Li, X., Ma, Y., Wright, J.: Robust Principal Component Analysis? (2009)

13. Holt, K.M.: Total Nuclear Variation and Jacobian Extensions of Total Variation for Vector Fields. IEEE Trans. Image Process. 23, 3975-3989 (2014). doi:10.1109/TIP.2014.2332397

14. Boyd, S., Parikh, N., Chu, E., Peleato, B., Eckstein, J.: Distributed Optimization and Statistical Learning via the Alternating Direction Method of Multipliers. Found. Trends® Mach. Learn. 3, 1-122 (2010). doi:10.1561/2200000016

15. Kowalski, M.: Sparse regression using mixed norms. Appl. Comput. Harmon. Anal. 27, 303-324 (2009). doi:10.1016/j.acha.2009.05.006

16. Rao, B.D., Kreutz-Delgado, K.: An affine scaling methodology for best basis selection. IEEE Trans. Signal Process. 47, 187-200 (1999). doi:10.1109/78.738251

17. Cai, J.-F., Candès, E.J., Shen, Z.: A Singular Value Thresholding Algorithm for Matrix Completion. SIAM J. Optim. 20, 1956-1982 (2010). doi:10.1137/080738970 
18. Duvauchelle, P., Freud, N., Kaftandjian, V., Babot, D.: Computer code to simulate X-ray imaging techniques. Nucl. Instruments Methods Phys. Res. Sect. B Beam Interact. with Mater. Atoms. 170, 245-258 (2000). doi:10.1016/S0168583X(00)00185-3

19. Saloman, E.B., Hubbell, J.H.: X-ray attenuation coefficients (total cross sections): Comparison of the experimental data base with the recommended values of Henke and the theoretical values of Scofield for energies between 0. 1-100 keV, https://www.osti.gov/scitech/biblio/6965777, (1986)

20. Vacca, R.: Regression Diagnostics - Identifying Influential Data and Sources of Collinearity. Wiley (1981)

21. Ding, Q., Niu, T., Zhang, X., Long, Y.: Image-domain multi-material decomposition for dual-energy CT based on correlation and sparsity of material images. (2017)

22. Zhang, Y., Xi, Y., Yang, Q., Cong, W.: Spectral CT Reconstruction With Image Sparsity and Spectral Mean. 2, 510523 (2016). doi:10.1109/TCI.2016.2609414

23. WANG, S.L., LIAO, L.Z.: Decomposition Method with a Variable Parameter for a Class of Monotone Variational Inequality Problems. J. Optim. Theory Appl. 109, 415-429 (2001). doi:10.1023/A:1017522623963 